\title{
Has Ivermectin Virus-Directed Effects against SARS-CoV-2? Rationalizing the Action of a Potential Multitarget Antiviral Agent
}

\author{
Antonio Francés-Monerris, ${ }^{1,2,{ }^{*}}$ Cristina García-Iriepa,,${ }^{3,4,}$ Isabel Iriepa, ${ }^{4,5}$ Cécilia Hognon, ${ }^{1}$ Tom Miclot, ${ }^{1,6}$ \\ Giampaolo Barone, ${ }^{6}$ Antonio Monari, ${ }^{1, *}$ and Marco Marazzi ${ }^{3,4,{ }^{*}}$ \\ 1 Université de Lorraine and CNRS, LPCT UMR 7019, F-54000 Nancy, France. \\ 2 Departament de Química Física, Universitat de València, 46100 Burjassot, Spain. \\ 3 Department of Analytical Chemistry, Physical Chemistry and Chemical Engineering, Universidad de Alcalá, Ctra. Madrid-Barcelona, Km 33,600, 28871 Alcalá \\ de Henares, Madrid, Spain. \\ 4 Chemical Research Institute "Andrés M. del Río" (IQAR), Universidad de Alcalá, 28871 Alcalá de Henares, Madrid, Spain. \\ 5 Department of Organic and Inorganic Chemistry, Universidad de Alcalá, Ctra. Madrid-Barcelona, Km 33,600, 28871 Alcalá de Henares, Madrid, Spain. \\ 6 Department of Biological, Chemical and Pharmaceutical Sciences and Technologies, Università degli Studi di Palermo, Viale delle Scienze, 90128 Palermo, \\ Italy. \\ *A. F.-M.: antonio.frances@uv.es, C. G.-I.: cristina.garciai@uah.es, A. M.: Antonio.monari@univ-lorraine.fr, M. M.: marco.marazzi@uah.es
}

Supporting information for this article is given via a link at the end of the document.

\begin{abstract}
The novel SARS-CoV-2 coronavirus is causing a devastating pandemic in 2020, threatening public health in many countries. An unprecedented rapid and global response has been set in motion to identify efficient antiviral agents against SARS-CoV-2, mostly relying on the repurposing of drugs presenting or not previously known antiviral activity. Ivermectin is an approved drug used as antiparasitic in humans and animals with well documented broad-spectrum antiviral properties that emerge from host-directed effects. Recent results reported by Wagstaff and coworkers (Antiviral Research 2020, 178, 104787) show a potent inhibition of SARS-CoV2 replication in vitro by ivermectin, and clinical trials with human volunteers have already started. However, the mode of action of ivermectin is still largely unknown, especially at the molecular level. Here, we employ advanced molecular dynamics simulations to assess the influence of ivermectin on several key viral protein targets, with the aim to reveal the molecular bases of antiviral mechanisms against SARS-CoV-2. Interestingly, we show that ivermectin could be regarded as a multitarget agent, inhibiting different viral functions. These include blocking the recognition by the SARS-CoV-2 Receptor Binding Domain (RBD) of the Angiotensin-Converting Enzyme 2 (ACE2), the interactions with the two viral proteases $3 \mathrm{CL}^{\text {pro }}$ and $\mathrm{PL}^{\text {pro, }}$ and the SARS Unique Domain (SUD) non-structural protein. Hence, the wide spectrum of actions involving i) the interference with cell infection, ii) the inhibition of viral replication, and iii) elusion of the host immune system, could point to an unprecedented synergy between host- and virus-directed effects explaining the high anti-SARS-CoV-2 activity observed for this compound.
\end{abstract}

\section{Introduction}

The outbreak of the respiratory coronavirus disease (COVID-19), caused by the novel SARS-CoV-2 coronavirus, has largely spread worldwide in 2020 constituting a major public health threath.[1-3] Despite the fact that the mortality rate of COVID-19 is estimated at $1 \%$, and that the majority of the infections evolves rather benignly, the possibility of severe respiratory syndromes, especially in aging patients or in subjects presenting comorbidities, requiring intensive care assistance, is at the base of the pressure imposed by SARS-CoV-2 infections on the public health systems. This is also aggravated by the high infectivity of SARS-CoV-2, that may result in uncontrolled bursts of infections that have required the implementation of severe social distancing measures and movement restrictions, including full lock-down, causing significant social and economic damage.

In this context of emergency, the international scientific community is reacting with rapid responses to enhance the knowledge of the virus's fundamental processes and hence facilitate the control of the pandemic. This includes a very rapid sequencing of SARS-CoV-2 genome, solving the structures of key viral proteins, modeling of the pandemic spread to assist public decision, and developing better detection tests,[4] vaccines,[5] and effective treatments.[6] Due to the urgency posed by the pandemic, an efficient and relatively fast strategy to obtain suitable therapeutic agents consists in exploring the antiSARS-CoV-2 activity of drugs already approved for human use, [7] in a drug repurposing framework. Since the safety profiles of the candidates are well known, only the antiviral efficacy remains to be assessed, thus strongly reducing the time required for clinical tests and approval.

A drug that has shown promising results is the broad-spectrum antiparasitic ivermectin (Figure 1), which has been shown to inhibit SARS-CoV-2 replication in cell cultures with an $\mathrm{IC}_{50}$ of $\sim 2$ $\mu M$.[8] In particular, the action of ivermectin results in $99.8 \%$ reduction of cell associated viral RNA in 24 hours, while a $~ 5000-$ fold decrease of the viral RNA content is observed after 48 hours with a maintaining of the effect at 72 hours. Recently, Yang et al[9] have shown that ivermectin is a host-directed agent, i.e. the main targets of the drug are not in the pathogen but in the host cell. This is coherent with the broad antiviral activity observed against HIV, influenza, dengue virus, Zika, and other flaviviruses.[10] Ivermectin inhibits the transport of viral proteins into cellular nuclei mediated by importin $\alpha / \beta 1$ carriers, an import process related to the suppression of the host immune response by impairing mRNA function.[9] This pathogen-independent mechanism suggests that only very low concentrations of ivermectin could be sufficient to control SARS-CoV-2 infection.[11] A recent report have questioned that the plasma concentrations of $5 \mu \mathrm{M}$ necessary to inhibit the virus can be reached in clinical conditions, suggesting alternative approaches such as inhaled formulations with high local concentrations or the synergy with other antiviral agents.[12] Overall, there is general consensus concerning a great potential of ivermectin in COVID-19 therapeutics, although further medical and pharmaceutical evidences are required to validate its use at a large scale. $[13,14]$ 
The mechanistic picture provided only by the host-directed effects might be, however, incomplete. Other possible pathogen-specific antiviral mechanisms remain unexplored and could be crucial for the potent antiviral activity of ivermectin against SARS-CoV-2. Some recent studies[15-17] have found interactions between SARS-CoV-2 proteins and ivermectin, even though the results are mostly based on docking methods while extensive molecular dynamics simulations, indispensable to fully characterize the interactions, are still lacking

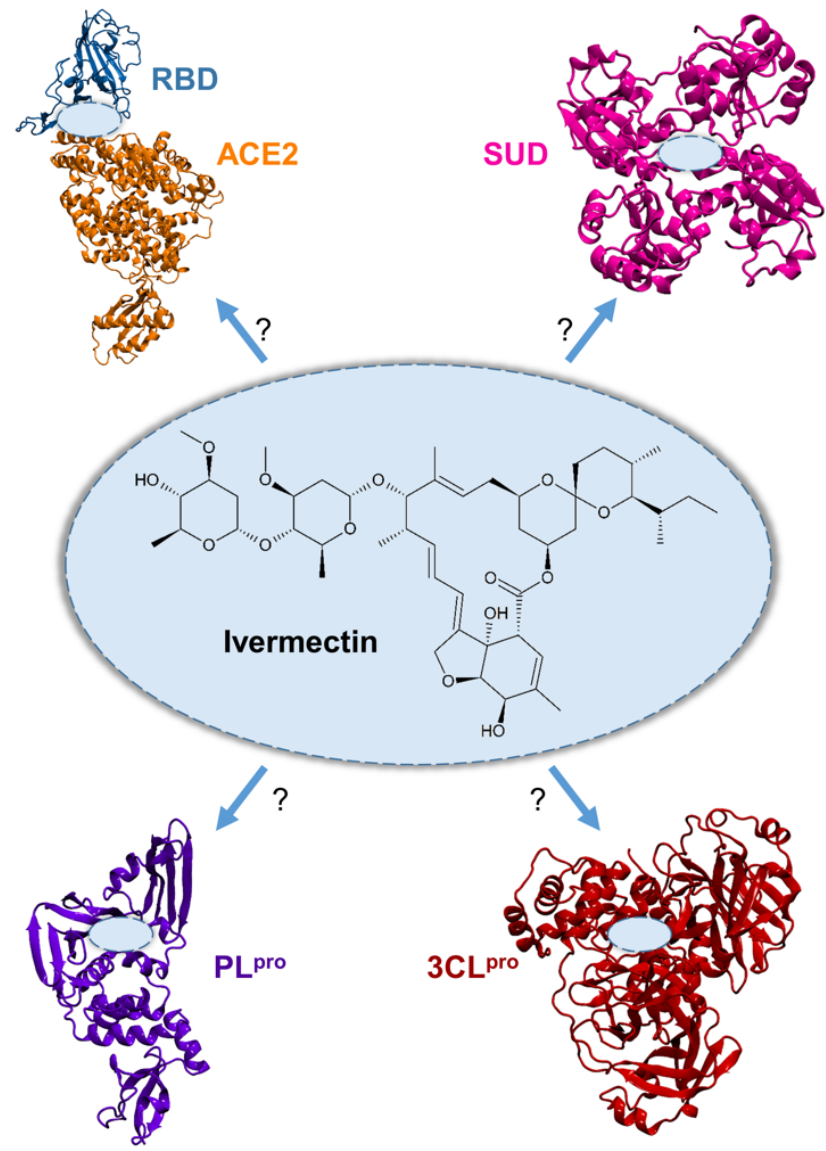

Figure 1. Chemical structure of ivermectin and schematic representation of the viral targets studied in this work.

In this work, we employ a combination of docking and state-ofthe-art all-atom molecular dynamics to explore the ability of ivermectin to interfere with SARS-CoV-2 viral structures (see Figure 1), and to quantify the dynamics of such interactions. In particular, we have focused to the following structures: $i$ ) the Angiotensin-Converting Enzyme 2 (ACE2)/Receptor Binding Domain (RBD) complex,[18-22] used by the virus to penetrate into the human cell; ii) the SARS Unique Domain (SUD) protein,[23-26] associated to the recognition of guanine quadruplexes sequences in mRNA to weaken the host immune response; iii) the main 3-chymotrypsin-like protease $3 \mathrm{CL}^{\text {pro }}$ (or $\left.\mathrm{M}^{\mathrm{pro}}\right),[27]$ used to catalyze the cleavage of specific peptide bonds in viral polyprotein precursors; $[28,29]$ and iv) papain-like protease $\left(P L^{\text {pro }}\right),[30]$ that cleaves the viral polyprotein at different sites than $\mathrm{M}^{\mathrm{pro}}$ and that may contribute to the immune system evasion by interfering with the cellular signaling pathways via its ubiquination sites.
As shall be discussed below in detail, our results show that ivermectin could be indeed regarded as a multitarget antiviral agent, potentially inhibiting all the above-mentioned targets to a certain extent. As a consequence, it may diminish cell infection by blockage of the ACE2/RBD complex as well as viral replication and maturation by interfering with viral proteases and SUD. These effects may complement the inhibition of nuclei carriers already observed in experiments.[9]

\section{Results and Discussion}

\section{Interaction with the $A C E 2 / R B D$ virus gate}

ACE2 is a glycoprotein with high affinity for sugar moieties,[31] therefore, glycosylated and polar small molecules such as ivermectin could compete with RBD in forming the typical hydrogen bonding network that mediates the protein-protein interaction. Our preliminary docking studies reveal that ivermectin forms stable aggregates with ACE2 (see Figure 2a). Diverse interaction hot spots are identified spanning different regions of the RBD recognition site, in particular, the interfaces $\alpha$ and $\beta$. Importantly, no specific interactions with the ACE2 natural catalytic active site, composed of the amino acid triad Arg708, Ser709, and Arg710,[32] are observed, ensuring the normal physiological function of ACE2 and thus avoiding extra side effects.

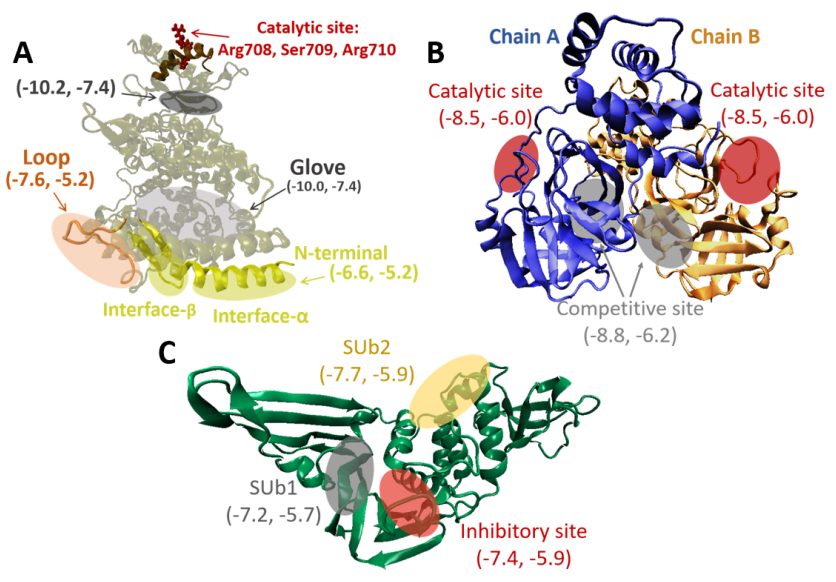

Figure 2. Binding energies resulting from molecular docking studies for each potential interacting site with ivermectin as inhibitory drug, including a) ACE2, b) $3 \mathrm{CL}^{\text {pro }}$ and c) PL pro.

The ability of ivermectin to disrupt the ACE2/RBD recognition has been subsequently quantified by means of all-atom MD simulations. It shall be noted that, for all initial pre-selected poses, the drug/ACE2 complex persists during all the simulation time (200 ns), indicating a high affinity between ivermectin and six ACE2 hot spots (four at interface- $\alpha$ and two at interface- $\beta$, as shown in Figure 3a). The binding is mainly mediated by hydrogen bonding and polar interactions allowed by the large number of available oxygen atoms in ivermectin (as alcohol, ether, and ester groups), in combination with its largely flexible molecular backbone, that allows an effective "covering" or "insertion" into the grooves formed by the ACE2 $\alpha$-helixes and $\beta$-sheets at the ACE2/RBD interface area. 
a)

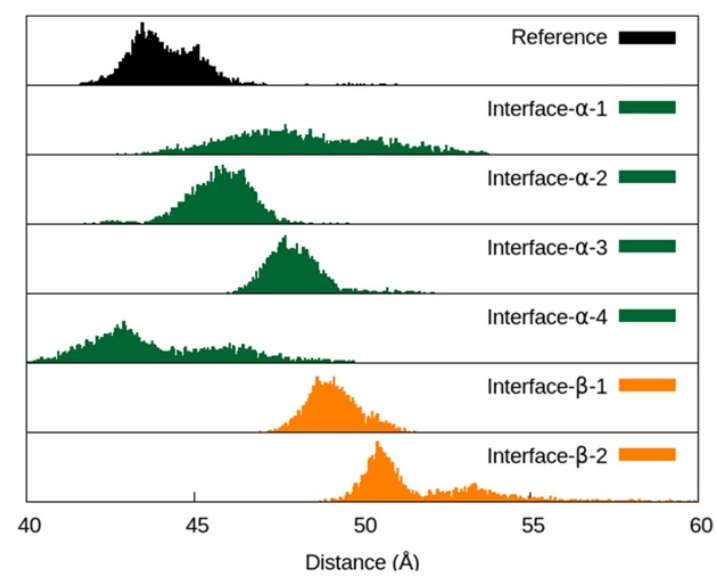

b)

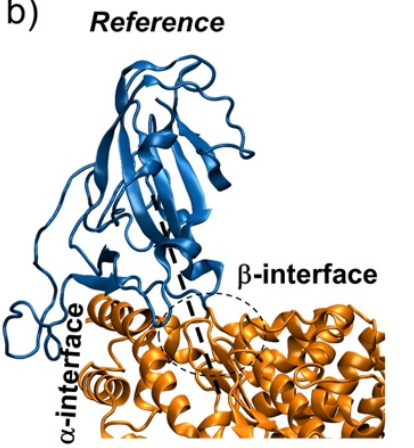

C) interface- $\beta-2$

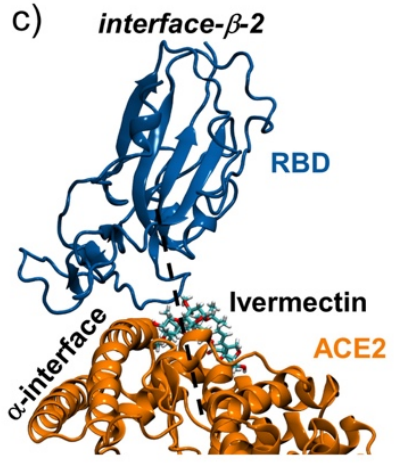

d)

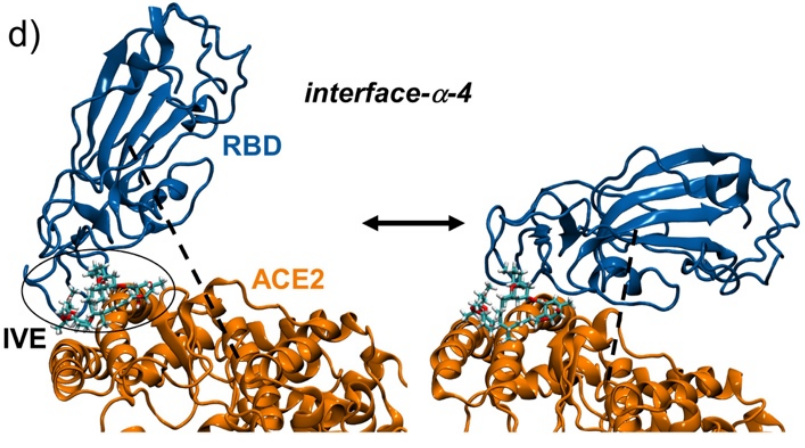

Figure 3. a) Distances between the ACE2 interface domain and the viral RBD in presence of ivermectin, measured with respect to the corresponding centers of mass (see text). b) Snapshot of the reference trajectory without ivermectin c) Ivermectin interacting at the interface- $\beta-2$ site. d) Bending of the RBD that leads to the "lateral" interaction with ACE2, with ivermectin complexed at the interface- $\alpha-4$ site, leading to shorter ACE2/RBD distances. Dashed black bars illustrate the distances represented in panel a).

Ivermectin inhibition effects have been quantified by measuring the distance between the centers of mass of the viral RBD and the host ACE2 structures, as described elsewhere.[22] From Figure $3 a$, it can be readily seen that five out of six poses shift the equilibrium distance of the complexes to significantly larger values, encompassing several Ångströms. This fact clearly indicates a strong destabilization of the ACE2/RBD recognition. The poses with the highest distance increase are those placed at the interface- $\beta$, i.e. the regions at the core of the ACE2/RBD interface (see Figures $3 b$ and $3 c$ ). Only the interface- $\alpha-4$ pose appears to actually decrease the protein/protein distances, being possibly interpreted as an improvement of the recognition. Nevertheless, the analysis of this trajectory reveals that the shorter distances between ACE2 and RBD centers of mass are not due to a more compact ACE2/RBD complex. Instead, the decrease in distance is mostly produced by the bending of the RBD protein and subsequent "lateral" or "side" interaction with ACE2, a type of interaction that most probably is not likely to promote viral infection (see Figure 3d). Indeed, such lateral interaction is not observed in the native system (reference, black bars in Figure 3a) since the RBD is not allowed to turn due to the strong ACE2/RBD interaction, that fixes the protein and its orientation. Finally, the presence of the drug also significantly broadens the distribution of the distances, further confirming the perturbation of the ACE2/RBD binding.

Inhibition of viral proteases $3 C L^{\text {pro }}$ and $P L^{\text {pro }}$

In addition to ACE2/RBD binding, we have evaluated the role of ivermectin on the inhibition of two proteases which are crucial for virus replication, namely the $3 \mathrm{CL}^{\text {pro }}$ and the $P L^{\text {pro }}$ proteases. It is known that the active form of $3 \mathrm{CL}^{\text {pro }}$ is a dimer, [33] presenting one catalytic site in each monomer. For this reason, we have used the dimer form as our model (see Computational Details section). Virtual docking results show that ivermectin interacts mainly with two sites of $3 \mathrm{CL}^{\text {pro: }}$ the inhibitory site, close to the catalytic site, and a competitive site (red and grey spots in Figure $2 \mathrm{~b}$, respectively), with similar binding energies $(6.2-8.8 \mathrm{kcal} / \mathrm{mol})$. It should be noted that such binding energies are slightly higher with respect to ACE2-ivermectin values at interfaces- $\alpha$ and $-\beta$ (5.2-6.6 $\mathrm{kcal} / \mathrm{mol})$.

Among them, the highest binding energies have been found for ivermectin interacting with its macrocyclic sixteen-membered lactone lying on the $3 \mathrm{CL}^{\text {pro }}$ surface, with the disaccharide moiety protruding within the pocket until reaching non-covalent contact with the catalytic dyad His41-Cys145 (Figure 4a). Alternatively, ivermectin can interact with the same macrocyclic lactone when the disaccharide moiety rotates, not reaching the catalytic dyad (Figure S1). Amongst the most promising docking poses including the above-mentioned features, four were selected to model the inhibition of two $3 \mathrm{CL}^{\text {pro }}$ dimers, considering the interaction of an ivermectin molecule in each binding site.

MD simulations of these two complexed dimeric systems have been performed to check the stability of the chosen poses and to analyze the ivermectin- $3 \mathrm{CL}^{\text {pro }}$ interaction along the simulation time. The simulations demonstrate that, in some cases, ivermectin does not bind stably. One simulation shows that ivermectin leaves the binding pocket after $78 \mathrm{~ns}$, later interacting with the external surface of $3 \mathrm{CL}^{\text {pro }}$, although the starting geometry had the disaccharide moiety in contact with the dyad (Figure S2a). Later, when the disaccharide moiety of ivermectin is away from the dyad, the simulation reveals that ivermectin rotates on the binding surface until reaching a different stable conformation with the disaccharide moiety now pointing inside the binding pocket (Figure S3). These findings suggest that the stability of ivermectin inside the $3 \mathrm{CL}^{\text {pro }}$ binding pocket is independent from the starting pose, while $3 \mathrm{CL}^{\text {pro }}$ fluctuations could drive different conformations leading to efficient inhibitions.

In two other cases, the interaction of ivermectin is stable along the simulation time, keeping the disaccharide moiety deep inside the binding pocket. A detailed analysis of the interactions, in terms of hydrogen-bonds and hydrophobic interactions, reveals that the disaccharide moiety interacts synergistically through direct and water-mediated hydrogen bonding, involving Thr26, His41, 
Asn142, His164, Glu166, Asp187, and Gln189 (Figure 4b), but also through hydrophobic interactions (Figure 4c).

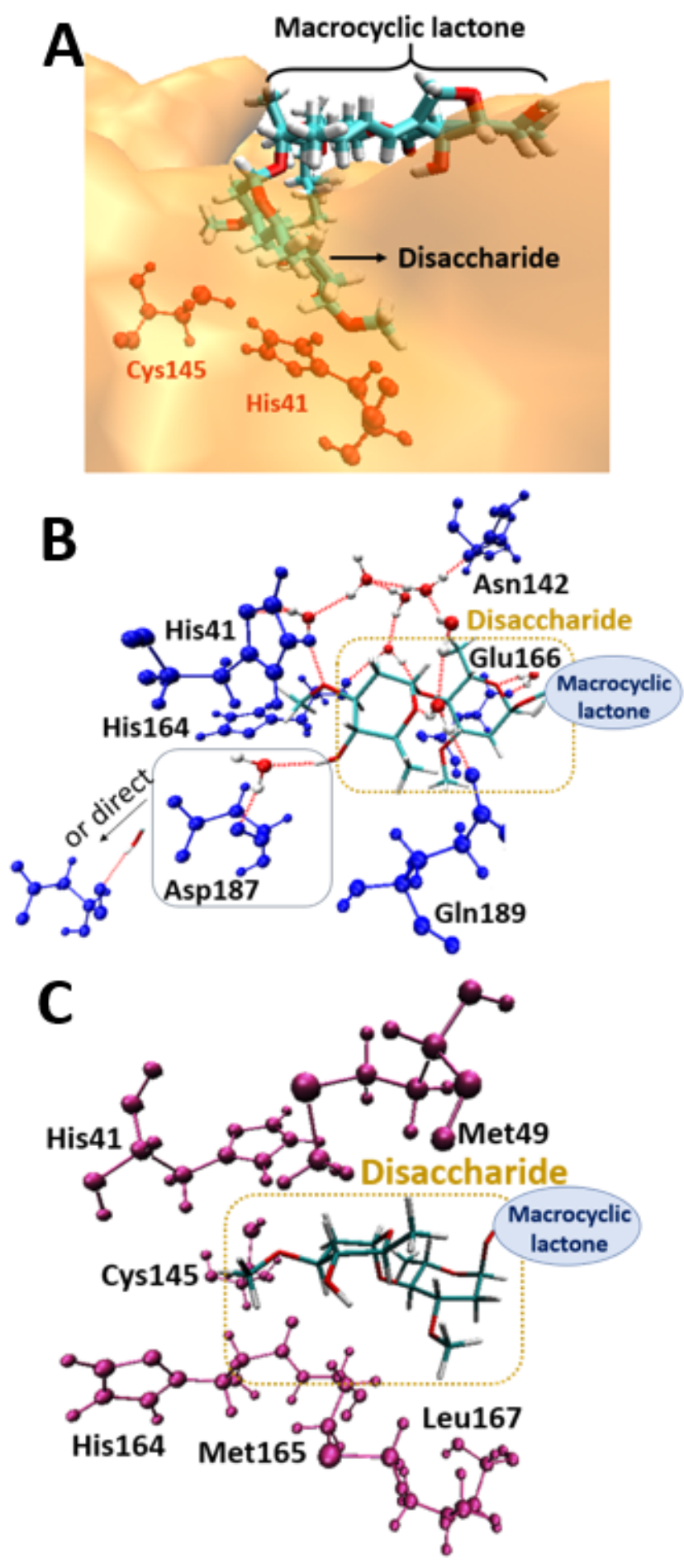

Figure 4. a) Most efficient binding interaction between ivermectin and $3 \mathrm{CL}^{\text {pro }}$, detailing its b) hydrogen-bonds and c) hydrophobic interactions.

Also, the macrocyclic lactone is stabilized through hydrogen bonding with Ser46, Glu47 and Gln189, and via hydrophobic interactions with Met49 and Ala191 (Figure S4). Hence, the interaction of ivermectin with the $3 \mathrm{CL}^{\text {pro }}$ binding site appears favorable to lead to catalytic inhibition as the drug interacts with the main subsites described for the enzyme binding pocket: $\mathrm{S} 1$ (conserved in all coronaviruses and comprising amino acids 140145 and 163-166), S2 (composed by amino acids 186-189 and side chains of His41, Met49 and Met165) and S4 (comprising amino acids 165, 167, 189, 190 and 192).[34-40] Moreover, the size and structure of ivermectin (macrocycle lactone and disaccharide) allows the interaction with the whole binding pocket, as compared to other proposed drugs whose interaction with some pocket subsites is missing or weaker.[36,37,40] Nevertheless, the large fluctuations observed for this protease, coupled to the inherently high flexibility of ivermectin, are expected to affect the inhibition efficiency, since a multiconformational complex equilibrium between bounded and unbounded ivermectin may also take place.

$\mathrm{PL}^{\text {pro }}$ has also been identified as crucial for virus replication. For this reason, we have evaluated the inhibition capacity of ivermectin against this enzyme. Differently from $3 \mathrm{CL}^{\text {pro, }}$, the monomeric form is the biologically active form for this enzyme.[41] Docking results revealed three main interaction sites with comparable binding energies: the inhibitory site and two ubiquitinlike (Ub) binding subsites, SUb1 and SUb2 (Figure 2c). Two MD simulations have been performed starting from two different ivermectin poses in the inhibitory site. One of these simulations is stable, showing a strong interaction of ivermectin inside the inhibitory site. In the other one, ivermectin moves out after ca. 100 ns (Figure S6). By analyzing in more detail the two simulations, we observe that the departure of ivermectin from the $\mathrm{PL}^{\text {pro }}$ surface is due to the opening of the flexible BL2 loop (also known as G267-G272 loop,[30,41] see Figure S6b). Indeed, when this loop keeps closed, the ivermectin binding mode is stable. In this latter case, ivermectin interacts through hydrogen-bonds and hydrophobic interactions with the inhibitory site. In particular, ivermectin interacts through direct and/or water-mediated hydrogen bonds (Asp165, Asn168 and GIn270) and through hydrophobic interactions (Cys112, Leu163, Pro248, Pro249, Tyr265 and Tyr269), similarly to $3 \mathrm{CL}^{\text {pro }}$ (Figure $5 \mathrm{~A}$ ). These types of interaction are in agreement with previously reported $\mathrm{PL}^{\text {pro }}$ inhibitors.[30,41-43]

Regarding the interaction of ivermectin with the SUb1 and SUb2 sites, one MD simulation for each case has been performed to ascertain the stability of the interaction. For SUb1, a stable interaction mode is found, with the disaccharide moiety lying mainly on top of the side chain of Arg167, while the macrocycle lactone is exposed to the solvent. This stable interaction arises from hydrogen bond interactions of the disaccharide moiety with Arg167 and Ser171 and hydrophobic interactions with Met209 and Pro248 (Figure 5b). By comparing these interactions with the ones reported for Ub inhibitors interacting in the Sub1 site, we can conclude that, although ivermectin is stable in this site, its position is slightly shifted towards the inhibitory site leading to a lower interaction, mainly driven by hydrogen-bond.[43,44] Analyzing the ivermectin-SUb2 simulation, ivermectin is shown to be unstable moving along the $\mathrm{PL}^{\text {pro }}$ surface between SUb1 and SUb2 until reaching a stable conformation at ca. 300 ns close to the SUb2 site (Figure S7b). In this case, the macrocyclic lactone is the moiety interacting with the $\mathrm{PL}^{\text {pro }}$ surface, whereas the disaccharide is exposed to the solvent (Figure $5 c$ ). In particular, ivermectin interacts through hydrogen bond interactions with His74, Thr75, Asp77, Tyr155, and Tyr172 and leads to hydrophobic interactions with Thr76, Pro130, Asn157, Tyr172, 
and His176. Comparing these bindings with the ones already reported for ubiquitin and ISG15 in the SUb2 domain, ivermectin interacts in the same region but with slightly different amino acids, probably due to its smaller size.[43,44] Hence, we can conclude that the interaction of ivermectin with the SUb domains of $\mathrm{PL}^{\text {pro }}$ is energetically favorable although in the case of SUb2 it is not persistent. All in all, these results suggest that ivermectin can both inhibit $\mathrm{PL}^{\text {pro }}$ and hamper the recognition of ubiquitin, helping the immune system to recover.
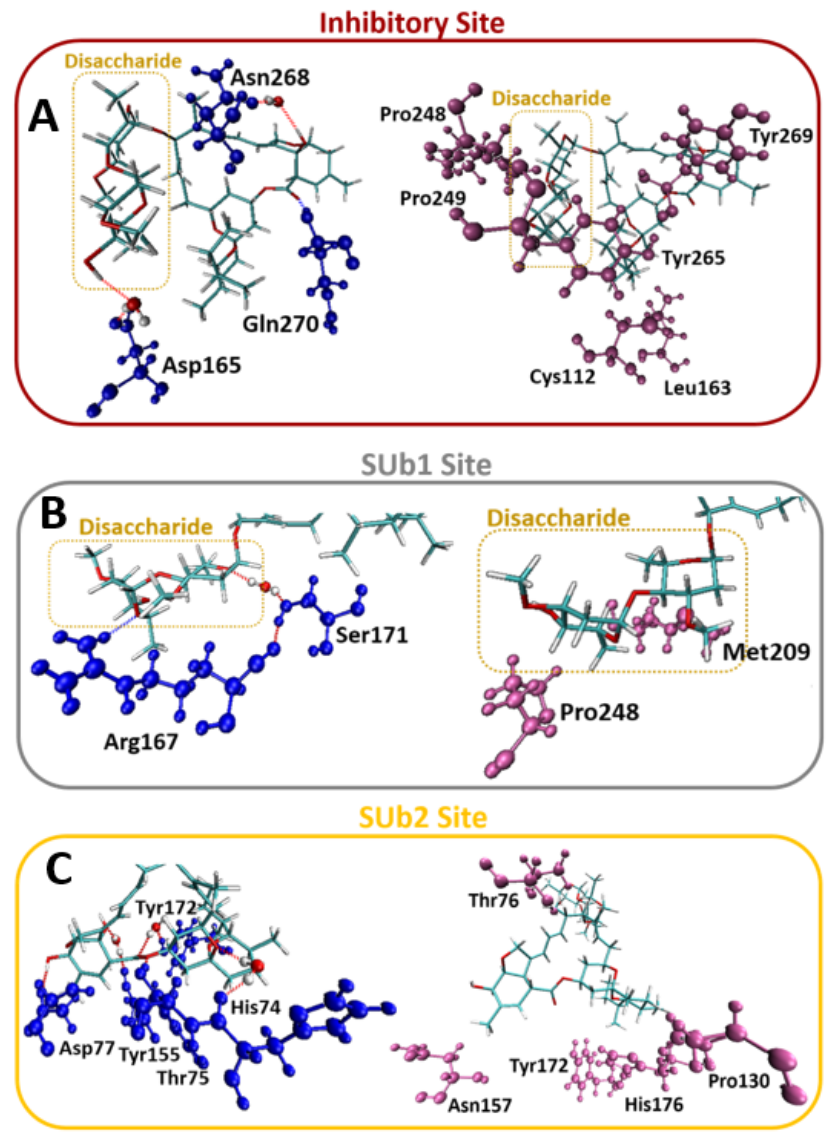

Figure 5. Hydrogen-bonds (left) and hydrophobic interactions (right) driving the binding modes between ivermectin and $\mathrm{PL}^{\text {pro }}$ at a) the inhibitory site, b) the SUb1 and c) the Sub2 sites.

Interaction with viral SUD and human G4 RNA

The SUD subunit of the SARS non-structural proteins have been deemed important for their capacity to highjack the host RNA, mainly via extensive electrostatic interactions with positive lysine residues. This in turn leads to an alteration of the protein transcription that helps SARS viruses to resist to the cellular immune response. MD simulations have recently revealed the atomistic details of the interaction.[26] It has been shown that SUD binds to human RNA in G-quadruplex (G4) arrangement and that the interaction between SUD and the G4 RNA helps maintaining the dimeric form of the viral protein, a prerequisite for its capacity to interfere with the immune response. In this respect, the possibility to directly interfere with the RNA recognition site of SUD may be regarded as a potential therapeutic strategy to diminish coronavirus resistance and its capacity of infection. Indeed, the inhibition of SUD activity could be achieved by small drugs aimed at occupy the G4 RNA interaction site, precluding the sequestration of human RNA. Another mode of action, more "indirect", could be the formation of stable aggregates between G4 RNA and the drug, thereby also precluding the formation of the SUD/RNA complex.

As reported in the $\mathrm{SI}$, the most stable complex between ivermectin and G4 RNA involves an interaction with the peripheral tetrads of the $\mathrm{G} 4$, while no stable groove binding mode has been identified. Since the main interaction between SUD and G4 involves electrostatic binding of the nucleic acid with the positive, lysinerich region of the protein, it is unlikely that the interaction with the G4 is sufficient to significantly perturb the nucleic acid binding to SUD. However, the fact that ivermectin binds to the peripheral tetrads of G4 RNA, could perturb the monomeric binding mode between RNA and SUD.[26] Even if the monomeric binding mode is not sufficient to maintain the compact and closed structure of SUD, as shown by Hognon et al.,[26] this mode can be used for the initial recruiting of nucleic acids, hence its perturbation should not be totally innocent.

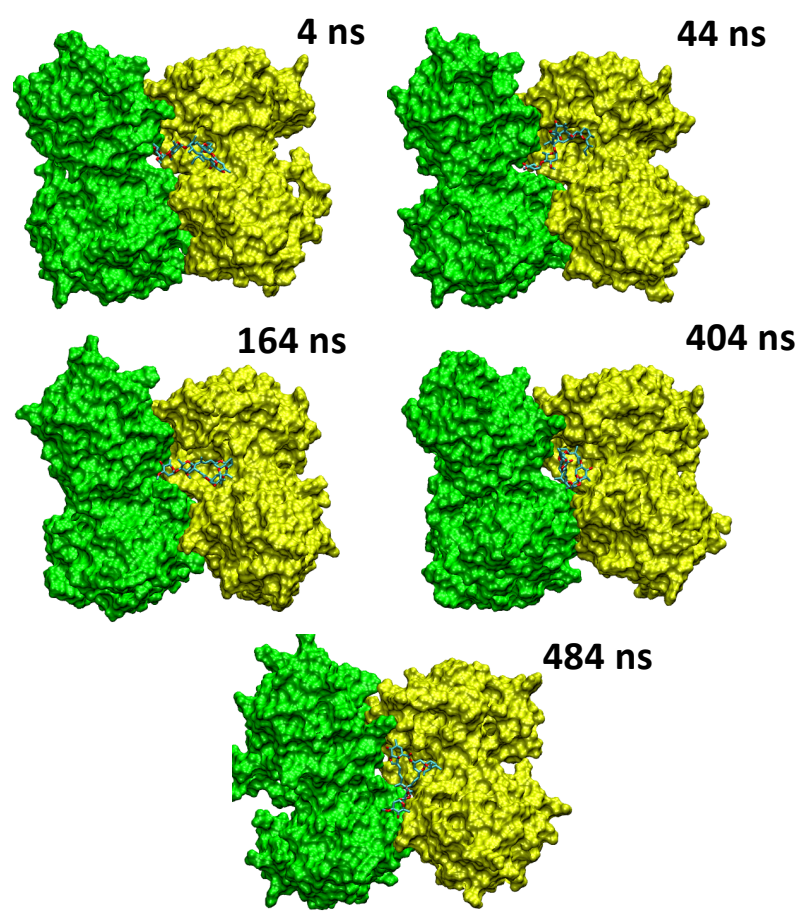

Figure 6. Representative snapshots extracted along the MD trajectory and depicting the SUD/ivermectin complex.

Conversely, a persistent occupation of the RNA recognition site of SUD by ivermectin is observed all along the MD trajectory, as pictorially represented in Figure 6. Although the ivermectin/SUD complex appears as stable, and the drug is consistently occupying the lysine rich region used to bind RNA, no specific and highly persistent interaction is evidenced at the molecular level. Instead, some labile hydrogen-bonds with the polar groups of ivermectin take place, although never persisting for more than some nanoseconds. As a consequence, the SUD/ivermectin complex seems to be mostly driven by hydrophobic and rather non-specific interactions. This fact also justifies the observation that, although the complex is persistent, ivermectin is characterized by a relatively high mobility resulting in rather 
complex sliding and rotation motions of the drug across the large interaction area, and by SUD conformational rearrangements between open and folded conformations. However, as previously pointed out, this high mobility does not break the drug/protein complex.

Hence, the results of the MD simulations indicate the possible occurrence of moderate interactions of ivermectin with SUD, in turn capable of perturbing the recognition and the association of the former protein with human G4 RNA, hence counteracting its capacity to favor the evasion from the host immune system. However, it shall be noted that, in light of the present results, SUD should be considered as a possible secondary target of ivermectin, complementing the more intense interactions with the RBD/ACE2 complex and the proteases, illustrating the multitarget character of ivermectin.

\section{Conclusion}

By using extensive MD simulations we have unraveled, at a molecular and atomic resolution, some possible mechanisms of action of ivermectin to counteract SARS-CoV-2 infection through competitive inhibition of several viral targets. In particular, we have shown that ivermectin can be characterized as a multitarget agent capable of interfering with different key steps of SARS-CoV2 multiplication cycle. These mechanisms could add to the known capabilities of ivermectin to block the transport of viral proteins into cellular nuclei mediated by importin $\alpha / \beta 1$ carriers.[9] Indeed, the impressive destabilization of the RBD/ACE2 complex in presence of ivermectin supports a direct inhibition of the SARSCoV-2 entry into the host cell. On the other hand, the inhibition of the active site of both $3 \mathrm{CL}^{\text {pro }}$ and $\mathrm{PL}^{\text {pro }}$ viral proteases could also contribute to inhibit the virus maturation after the infection. Finally, ivermectin might also impede the ubiquination of $\mathrm{PL}^{\text {pro }}$ and the interaction between the viral SUD and human RNA, that are recognized as mechanisms used by coronaviruses to weaken the host immune defenses and hence assure the virus survival.

The present results are important in the development of rational drug design or drug-repurposing strategies in the fight against COVID-19. Indeed, ivermectin represents an ideal case study, highlighting the different viral or host protein sites that should be targeted to inhibit clear viral functions. Thus, this work provides evidence of an unprecedented synergy between host- and viraldirected mechanisms of action for ivermectin, a first step in the exploration of this dual mechanism. Nevertheless, further experimental and theoretical studies are encouraged to confirm this novel concept.

\section{Computational Details}

\section{Preparation of the systems}

Discovery Studio 2.1 program was used to add hydrogen atoms and assign bond orders, hybridization and charges to ACE2, extracted from PDB ID 6M17.[21] Three-dimensional coordinates of the $3 \mathrm{CL}^{\text {pro }}$ were retrieved from the PDB ID 6 LU7 (dimer form). The PL pro protein model (monomer form) has been extracted the PDB ID 6W9C. We have selected chain $\mathrm{C}$ as it is the most complete one. For the $\mathrm{Zn}$ finger (cysteine residues interacting with the $\mathrm{Zn}$ atom), we have used a non-bonded strategy to build the parameters, as recently documented in the literature.[45] In the case of SUD the system, i.e. the protein (PDB ID 2W2G) and the RNA (PDB ID
$18 \mathrm{JG})$, have been prepared coherently with the procedure recently used by us.[26]

\section{Docking}

Flexible drug docking studies have been performed using the Autodock Vina software[46] to determine meaningful binding poses of ivermectin. Prior to virtual screening, the 3D geometry of ivermectin was built with the Discovery Studio 2.1 program.

For the ACE2/RBD system, the grid center coordinates were $x=154.410$, $y=131.537, z=213.591$ and the size coordinates were $x=100, y=100$, $z=100$ with grid points separated by $1 \AA$. For $3 \mathrm{CL}^{\text {pro }}$ the grid center coordinates were $x=53.065, y=161.664, z=26.000$ and the size coordinates were $x=24, y=34, z=38$ with grid points separated by $1 \AA$. For PL pro the grid center coordinates were $x=-55.400, y=10.700, z=$ 24.020 and the size coordinates were $x=84, y=82, z=86$ with grid points separated by $1 \AA$

\section{Molecular dynamics simulations}

High-level equilibrium molecular dynamics (MD) have been employed on the pre-selected configurations to quantify the biological activity of ivermectin towards a given viral structure, using the NAMD program[47] in combination with the Amber99SB force field,[48-50] including the bsc1 corrections,[51] to describe proteins and RNA, and TIP3P[52] parameters to treat water molecules. Meanwhile, the force field of ivermectin was parameterized through the GAFF procedure.[53]

The initial structures were minimized applying 8000 steps of the NAMD conjugate gradient and line search algorithm keeping the protein backbone frozen. The constraints were gradually relaxed during $36 \mathrm{ps}$, reaching a final temperature of $300 \mathrm{~K}$ at the NPT ensemble. MD production runs were simulated at $300 \mathrm{~K}$ and $1 \mathrm{~atm}$, with total simulation times of 0.2 (RBD/ACE2) and $0.5 \mu$ s (proteases, SUD/RNA system). The hydrogen mass repartition (HMR) method[54] has been applied in all systems, allowing a timestep of $4 \mathrm{fs}$. The pressure was set to $1 \mathrm{~atm}$ and maintained constant using the Nosé-Hoover Langevin piston pressure control, while temperature conservation was ensured through Langevin dynamics. All MD simulations were conducted using periodic boundary conditions and the particle mesh-Ewald (PME) method with a cutoff of $9.0 \AA$. Results have been analyzed through VMD.[55] In the case of RBD/ACE2 simulations, the RBD was initially separated 5 Angstrom from the ACE2 in order to include ivermectin at the interaction sites revealed by the docking studies, also in the reference run without ivermectin.

\section{Acknowledgements}

Support from the Universite de Lorraine and French CNRS is gratefully acknowledged. A.F.-M. is grateful to Generalitat Valenciana and the European Social Fund (contract APOSTD/2019/149, project GV/2020/226) and the Ministerio de Ciencia e Innovación (project CTQ2017-87054-C2-2-P) for financial support. C.G.-I. and M.M. are grateful to the Ministerio de Economía y Competitividad, Spain (CTQ2016-80600-P). French CNRS and GENCI IDRIS national computing center are acknowledged for graciously providing access to computational resources in the framework of the special COVID-19 mobilization under the project "Seek\&Destroy". Part of the calculations have been performed on the LPCT local computing cluster, on the regional ExpLor center in the frame of the project "Dancing Under the Light", and on the local computing cluster of the "Reactivity and Molecular Structure Group" at the Universidad de Alcalá. 
Keywords: COVID-19 - ACE2 - SARS Unique Domain • Proteases $•$ Molecular Dynamics

1. Tu, Y.-F., Chien, C.-S., Yarmishyn, A.A., Lin, Y.-Y., Luo, Y.-H., Lin, Y.-T., Lai, W.-Y., Yang, D.-M., Chou, S.-J., and Yang, Y.-P. (2020) A Review of SARS-CoV-2 and the Ongoing Clinical Trials. Int. J. Mol. Sci., 21 (7), 2657. Watkins, J. (2020) Preventing a covid-19 pandemic. BMJ, 368, doi 10.1136/bmj.m810.

3. Bedford, J., Enria, D., Giesecke, J., Heymann, D.L., Ihekweazu, C., Kobinger, G., Lane, H.C., Memish, Z., Oh, M. don, Sall, A.A., Schuchat, A., Ungchusak, K., and Wieler, L.H. (2020) COVID-19: towards controlling of a pandemic. Lancet, 395 (10229), 1015-1018. Kilic, T., Weissleder, R., and Lee, H. (2020) Molecular and immunological diagnostic tests of COVID-19 - current status and challenges. iScience, 101406.

Folegatti, P.M., Ewer, K.J., Aley, P.K., Angus, B., Becker, S., BelijRammerstorfer, S., Bellamy, D., Bibi, S., Bittaye, M., and Clutterbuck, E.A. (2020) Safety and immunogenicity of the ChAdOx1 $\mathrm{nCoV}-19$ vaccine against SARS-CoV-2: a preliminary report of a phase $1 / 2$, single-blind, randomised controlled trial. Lancet.

6. Florindo, H.F., Kleiner, R., Vaskovich-Koubi, D., Acúrcio, R.C., Carreira, B., Yeini, E., Tiram, G., Liubomirski, Y., and SatchiFainaro, R. (2020) Immune-mediated approaches against COVID19. Nat. Nanotechnol.

Gordon, D.E., Jang, G.M., Bouhaddou, M., Xu, J., Obernier, K., O'Meara, M.J., Guo, J.Z., Swaney, D.L., Tummino, T.A., Huettenhain, R., Kaake, R.M., Richards, A.L., Tutuncuoglu, B., Foussard, H., Batra, J., Haas, K., Modak, M., Kim, M., Haas, P., Polacco, B.J., Braberg, H., Fabius, J.M., Eckhardt, M., Soucheray, M., Bennett, M.J., Cakir, M., McGregor, M.J., Li, Q., Naing, Z.Z.C., Zhou, Y., Peng, S., Kirby, I.T., Melnyk, J.E., Chorba, J.S., Lou, K., Dai, S.A., Shen, W., Shi, Y., Zhang, Z., Barrio-Hernandez, I., Memon, D., Hernandez-Armenta, C., Mathy, C.J.P., Perica, T., Pilla, K.B., Ganesan, S.J., Saltzberg, D.J., Ramachandran, R., Liu, X., Rosenthal, S.B., Calviello, L., Venkataramanan, S., Liboy-Lugo, J., Lin, Y., Wankowicz, S.A., Bohn, M., Sharp, P.P., Trenker, R., Young, J.M., Cavero, D.A., Hiatt, J., Roth, T.L., Rathore, U., Subramanian, A., Noack, J., Hubert, M., Roesch, F., Vallet, T., Meyer, B., White, K.M., Miorin, L., Rosenberg, O.S., Verba, K.A., Agard, D., Ott, M., Emerman, M., Ruggero, D., García-Sastre, A., Jura, N., von Zastrow, M., Taunton, J., Ashworth, A., Schwartz, O., Vignuzzi, M., d'Enfert, C., Mukherjee, S., Jacobson, M., Malik, H.S., Fujimori, D.G., Ideker, T., Craik, C.S., Floor, S., Fraser, J.S., Gross, J., Sali, A., Kortemme, T., Beltrao, P., Shokat, K., Shoichet, B.K., and Krogan, N.J. (2020) A SARS-CoV-2-Human Protein-Protein Interaction Map Reveals Drug Targets and Potential DrugRepurposing. bioRxiv, 2020.03.22.002386.

8. Caly, L., Druce, J.D., Catton, M.G., Jans, D.A., and Wagstaff, K.M. (2020) The FDA-approved drug ivermectin inhibits the replication of SARS-CoV-2 in vitro. Antiviral Res., 178, 104787.

9. Yang, S.N.Y., Atkinson, S.C., Wang, C., Lee, A., Bogoyevitch, M.A., Borg, N.A., and Jans, D.A. (2020) The broad spectrum antiviral ivermectin targets the host nuclear transport importin $\alpha / \beta 1$ heterodimer. Antiviral Res., 177, 104760.
B.R., Sah, R., Bonilla-Aldana, D.K., Rodriguez-Morales, A.J., and Leblebicioglu, H. (2020) Ivermectin, a new candidate therapeutic against SARS-CoV-2/COVID-19. Ann. Clin. Microbiol. Antimicrob., 19 (1), 23.

11. Bray, M., Rayner, C., Noël, F., Jans, D., and Wagstaff, K. (2020) Ivermectin and COVID-19: a report in Antiviral Research, widespread interest, an FDA warning, two letters to the editor and the authors' responses. Antiviral Res., 104805.

12. Peña-Silva, R., Duffull, S.B., Steer, A.C., Jaramillo-Rincon, S.X., Gwee, A., and Zhu, X. (2020) Pharmacokinetic considerations on the repurposing of ivermectin for treatment of COVID-19. Br. J. Clin. Pharmacol., n/a (n/a).

13. Kumar, B.S., Jeyaraman, M., Jain, R., and Anudeep, T.C. (2020) A Wonder Drug in the Arsenal against COVID-19: Medication Evidence from Ivermectin. J. Adv. Med. Med. Res., 30-37.

14. Rajter, J.C., Sherman, M., Fatteh, N., Vogel, F., Sacks, J., and Rajter, J.-J. (2020) ICON (Ivermectin in COvid Nineteen) study: Use of Ivermectin is Associated with Lower Mortality in Hospitalized Patients with COVID19. medRxiv, 2020.06.06.20124461.

15. LEHRER, S., and RHEINSTEIN, P.H. Ivermectin Docks to the SARS-CoV-2 Spike Receptor-binding Domain Attached to ACE2.

16. Maurya, D.K. (2020) A Combination of Ivermectin and Doxycycline Possibly Blocks the Viral Entry and Modulate the Innate Immune Response in COVID-19 Patients.

17. Dasgupta, J., Sen, U., Bakshi, A., Dasgupta, A., Manna, K., Saha, C., De, R.K., Mukhopadhyay, S., and Bhattacharyya, N.P. (2020) Nsp7 and Spike Glycoprotein of SARS-CoV-2 are envisaged as Potential Targets of Vitamin D and Ivermectin.

18. Wang, Q., Zhang, Y., Wu, L., Niu, S., Song, C., Zhang, Z., Lu, G., Qiao, C., Hu, Y., Yuen, K.Y., Wang, Q., Zhou, H., Yan, J., and Qi, J. (2020) Structural and Functional Basis of SARS-CoV-2 Entry by Using Human ACE2. Cell, 181 (4), 894-904.e9.

19. Shang, J., Ye, G., Shi, K., Wan, Y., Luo, C., Aihara, H., Geng, Q., Auerbach, A., and Li, F. (2020) Structural basis of receptor recognition by SARS-CoV-2. Nature.

20. Lan, J., Ge, J., Yu, J., Shan, S., Zhou, H., Fan, S., Zhang, Q., Shi, X., Wang, Q., Zhang, L., and Wang, X. (2020) Structure of the SARS-CoV-2 spike receptor-binding domain bound to the ACE2 receptor. Nature, 581 (7807), 215-220.

21. Yan, R., Zhang, Y., Li, Y., Xia, L., Guo, Y., and Zhou, Q. (2020) Structural basis for the recognition of SARS-CoV-2 by full-length human ACE2. Science (80-. ).

22. Garcia-Iriepa, C., Hognon, C., Francés-Monerris, A., Iriepa, I., Miclot, T., Barone, G., Monari, A., and Marazzi, M. (2020) Thermodynamics of the Interaction Between SARS-CoV-2 Spike Protein and Human ACE2 Receptor. Effects of Possible Ligands.

23. Lei, J., Kusov, Y., and Hilgenfeld, R. (2018) Nsp3 of coronaviruses: Structures and functions of a large multi-domain protein. Antiviral Res., 149, 58-74.

24. Kusov, Y., Tan, J., Alvarez, E., Enjuanes, L., and Hilgenfeld, R. (2015) A G-quadruplex-binding macrodomain within the "SARSunique domain" is essential for the activity of the SARS-coronavirus replication-transcription complex. Virology, 484, 313-322.

25. Tan, J., Vonrhein, C., Smart, O.S., Bricogne, G., Bollati, M., Kusov, Y., Hansen, G., Mesters, J.R., Schmidt, C.L., and Hilgenfeld, R. (2009) The SARS-Unique Domain (SUD) of SARS coronavirus contains two macrodomains that bind G-quadruplexes. PLoS 
Pathog., 5 (5), e1000428.

Hognon, C., Miclot, T., Iriepa, C.G., Francés-Monerris, A. Grandemange, S., Terenzi, A., Marazzi, M., Barone, G., and Monari, A. (2020) Role of RNA Guanine Quadruplexes in Favoring the Dimerization of SARS Unique Domain in Coronaviruses. J. Phys. Chem. Lett., 11, 5661-5667.

27. Zhang, L., Lin, D., Sun, X., Curth, U., Drosten, C., Sauerhering, L., Becker, S., Rox, K., and Hilgenfeld, R. (2020) Crystal structure of SARS-CoV-2 main protease provides a basis for design of improved a-ketoamide inhibitors. Science (80-. )., 368 (6489), 409-412. Ramos-Guzmán, C.A., Ruiz-Pernía, J.J., and Tuñón, I. (2020) Unraveling the SARS-CoV-2 Main Protease Mechanism Using Multiscale DFT/MM Methods. chemRxiv.

29. Świderek, K., and Moliner, V. (2020) Revealing the molecular mechanisms of proteolysis of SARS-CoV-2 Mpro by QM/MM computational methods. Chem. Sci.

30. Ratia, K., Pegan, S., Takayama, J., Sleeman, K., Coughlin, M., Baliji, S., Chaudhuri, R., Fu, W., Prabhakar, B.S., Johnson, M.E., Baker, S.C., Ghosh, A.K., and Mesecar, A.D. (2008) A noncovalent class of papain-like protease/deubiquitinase inhibitors blocks SARS virus replication. Proc. Natl. Acad. Sci., 105 (42), 16119 LP - 16124

31. Prabakaran, P., Xiao, X., and Dimitrov, D.S. (2004) A model of the ACE2 structure and function as a SARS-CoV receptor. Biochem. Biophys. Res. Commun.

32. Lai, Z.W., Hanchapola, I., Steer, D.L., and Smith, A.I. (2011) Angiotensin-converting enzyme 2 ectodomain shedding cleavagesite identification: Determinants and constraints. Biochemistry.

33. Yang, H., Yang, M., Ding, Y., Liu, Y., Lou, Z., Zhou, Z., Sun, L., Mo, L., Ye, S., Pang, H., Gao, G.F., Anand, K., Bartlam, M., Hilgenfeld, R., and Rao, Z. (2003) The crystal structures of severe acute respiratory syndrome virus main protease and its complex with an inhibitor. Proc. Natl. Acad. Sci. U. S. A.

34. Mahanta, S., Chowdhury, P., Gogoi, N., Goswami, N., Borah, D., Kumar, R., Chetia, D., Borah, P., Buragohain, A.K., and Gogoi, B. (2020) Potential anti-viral activity of approved repurposed drug against main protease of SARS-CoV-2: an in silico based approach. J. Biomol. Struct. Dyn.

35. Macchiagodena, M., Pagliai, M., and Procacci, P. (2020) Identification of potential binders of the main protease 3CLpro of the COVID-19 via structure-based ligand design and molecular modeling. Chem. Phys. Lett.

36. Islam, R., Parves, R., Paul, A.S., Uddin, N., Rahman, M.S., Mamun, A. Al, Hossain, M.N., Ali, M.A., and Halim, M.A. (2020) A Molecular Modeling Approach to Identify Effective Antiviral Phytochemicals against the Main Protease of SARS-CoV-2. J. Biomol. Struct. Dyn. Huynh, T., Wang, H., and Luan, B. (2020) In Silico Exploration of Molecular Mechanism of Clinically Oriented Drugs for Possibly Inhibiting SARS-CoV-2's Main Protease. J. Phys. Chem. Lett.

38. Elmezayen, A.D., Al-Obaidi, A., Şahin, A.T., and Yelekçi, K. (2020) Drug repurposing for coronavirus (COVID-19): in silico screening of known drugs against coronavirus $3 \mathrm{CL}$ hydrolase and protease enzymes. J. Biomol. Struct. Dyn.

39. Sk, M.F., Roy, R., Jonniya, N.A., Poddar, S., and Kar, P. (2020) Elucidating biophysical basis of binding of inhibitors to SARS-CoV-2 main protease by using molecular dynamics simulations and free energy calculations. J. Biomol. Struct. Dyn.
Y.-J., Chinnasamy, S., and Wei, D.-Q. (2020) Marine natural compounds as potents inhibitors against the main protease of SARS-CoV-2. A molecular dynamic study. J. Biomol. Struct. Dyn.

41. Báez-Santos, Y.M., St. John, S.E., and Mesecar, A.D. (2015) The SARS-coronavirus papain-like protease: Structure, function and inhibition by designed antiviral compounds. Antiviral Res.

42. Báez-Santos, Y.M., Mielech, A.M., Deng, X., Baker, S., and Mesecar, A.D. (2014) Catalytic function and substrate specificity of the papain-like protease domain of nsp3 from the Middle East respiratory syndrome coronavirus. J. Virol., 88 (21), 12511-12527.

43. Ratia, K., Kilianski, A., Baez-Santos, Y.M., Baker, S.C., and Mesecar, A. (2014) Structural basis for the ubiquitin-linkage specificity and deISGylating activity of SARS-CoV papain-like protease. PLoS Pathog, 10 (5), e1004113.

44. Békés, M., Ekkebus, R., Ovaa, H., Huang, T.T., and Lima, C.D. (2016) Recognition of Lys48-linked di-ubiquitin and deubiquitinating activities of the SARS coronavirus papain-like protease. Mol. Cell, 62 (4), 572-585.

45. Macchiagodena, M., Pagliai, M., Andreini, C., Rosato, A., and Procacci, P. (2019) Upgrading and Validation of the AMBER Force Field for Histidine and Cysteine Zinc(II)-Binding Residues in Sites with Four Protein Ligands. J. Chem. Inf. Model., 59 (9), 3803-3816.

46. Trott, O., and Olson, A.J. (2009) AutoDock Vina: Improving the speed and accuracy of docking with a new scoring function, efficient optimization, and multithreading. J. Comput. Chem.

47. Phillips, J.C., Braun, R., Wang, W., Gumbart, J., Tajkhorshid, E., Villa, E., Chipot, C., Skeel, R.D., Kalé, L., and Schulten, K. (2005) Scalable molecular dynamics with NAMD. J. Comput. Chem., 26 (16), 1781-1802.

48. Maier, J.A., Martinez, C., Kasavajhala, K., Wickstrom, L., Hauser, K.E., and Simmerling, C. (2015) ff14SB: Improving the Accuracy of Protein Side Chain and Backbone Parameters from ff99SB. J. Chem. Theory Comput., 11 (8), 3696-3713.

49. Galindo-Murillo, R., Robertson, J.C., Zgarbová, M., Šponer, J., Otyepka, M., Jurečka, P., and Cheatham, T.E. (2016) Assessing the Current State of Amber Force Field Modifications for DNA. J. Chem. Theory Comput., 12 (8), 4114-4127.

50. Zgarbová, M., Šponer, J., Otyepka, M., Cheatham, T.E., GalindoMurillo, R., and Jurečka, P. (2015) Refinement of the SugarPhosphate Backbone Torsion Beta for AMBER Force Fields Improves the Description of Z- and B-DNA. J. Chem. Theory Comput., 11 (12), 5723-5736.

51. Ivani, I., Dans, P.D., Noy, A., Pérez, A., Faustino, I., Hospital, A., Walther, J., Andrio, P., Goñi, R., Balaceanu, A., Portella, G., Battistini, F., Gelpí, J.L., González, C., Vendruscolo, M., Laughton, C.A., Harris, S.A., Case, D.A., and Orozco, M. (2016) PARMBSC1: A refined force-field for DNA simulations. Nat. Methods, 13 (1), 5558.

52. Jorgensen, W.L., Chandrasekhar, J., Madura, J.D., Impey, R.W., and Klein, M.L. (1983) Comparison of simple potential functions for simulating liquid water. J. Chem. Phys., 79 (2), 926-935.

53. Wang, J.M., Wolf, R.M., Caldwell, J.W., Kollman, P.A., and Case, D.A. (2004) Development and testing of a general amber force field. J. Comput. Chem., 25 (9), 1157-1174.

54. Hopkins, C.W., Le Grand, S., Walker, R.C., and Roitberg, A.E. (2015) Long-time-step molecular dynamics through hydrogen mass repartitioning. J. Chem. Theory Comput., 11 (4), 1864-1874. 


\section{Entry for the Table of Contents}

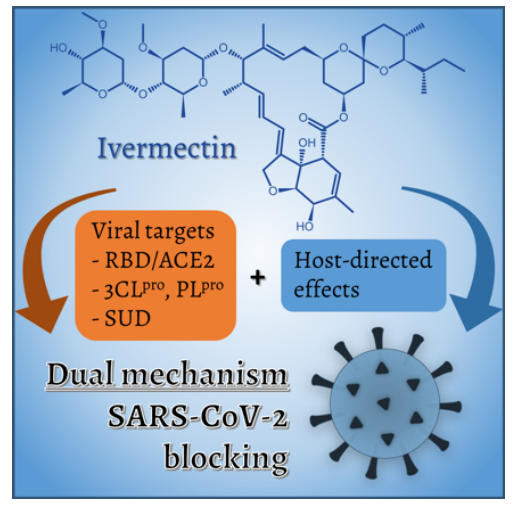

Molecular dynamics reveal the interaction hotspots between the antiparasitic drug ivermectin and several SARS-CoV-2 structures. These findings suggest a novel dual mechanism of action for this drug, in which both host-directed and virus-directe effects act synergistically to stop SARS-CoV-2 infection.

Institute and/or researcher Twitter usernames: @AntonioMonari @baronegiampaolo @RESMOL_UAH a 